\title{
On the Flag Curvature of Finsler Metrics of Scalar Curvature*
}

\author{
Xinyue Chen ${ }^{\dagger}$ Xiaohuan Mo ${ }^{\ddagger}$ and Zhongmin Shen
}

March 15, 2003

\begin{abstract}
The flag curvature of a Finsler metric is called a Riemannian quantity because it is an extension of sectional curvature in Riemannian geometry. In Finsler geometry, there are several non-Riemannian quantities such as the (mean) Cartan torsion, the (mean) Landsberg curvature and the Scurvature, which all vanish for Riemannian metrics. It is important to understand the geometric meanings of these quantities. In this paper, we study Finsler metrics of scalar curvature (i.e., the flag curvature is a scalar function on the slit tangent bundle) and partially determine the flag curvature when certain non-Riemannian quantities are isotropic. Using the obtained formula for the flag curvature, we classify locally projectively flat Randers metrics with isotropic S-curvature.
\end{abstract}

\section{Introduction}

Finsler metrics arise naturally from many areas of mathematics as well as natural science. For example, the navigation problem in a Riemannian space gives rise to a lots of interesting Finsler metrics with special geometric properties 28, 29] 30. In Finsler geometry, we study not only the shape of a space, but also the "color" of the space on an infinitesimal scale. The Riemannian quantity (such as the flag curvature) describes the shape of a space, while non-Riemannian quantities describes the "color" of the space.

For a Finsler manifold $(M, F)$, the flag curvature $\mathbf{K}=\mathbf{K}(P, y)$ is a function of tangent planes $P=\operatorname{span}\{y, v\} \subset T_{x} M$ and directions $y \in P \backslash\{0\}$. This quantity tells us how curved the space is at a point. If $F$ is Riemannian, $\mathbf{K}=$ $\mathbf{K}(P)$ is independent of $y \in P \backslash\{0\}$, $\mathbf{K}$ being called the sectional curvature in Riemannian geometry. A Finsler metric $F$ is said to be of scalar curvature if the flag curvature $\mathbf{K}=\mathbf{K}(x, y)$ is a scalar function on the slit tangent bundle

\footnotetext{
*2000 Mathematics Subject Classification: Primary 53B40, 53C60

${ }^{\dagger}$ supported by the National Natural Science Foundation of China (10171117)

¥supported by the National Natural Science Foundation of China (10171002)
} 
$T M \backslash\{0\}$. Clearly, a Riemannian metric is of scalar curvature if and only if $\mathbf{K}=\mathbf{K}(x)$ is a scalar function on $M$ (which is a constant in dimension $n>2$ by the Schur lemma). There are lots of non-Riemannian Finsler metrics of scalar curvature. One of the important problems in Finsler geometry is to study and characterize Finsler metrics of scalar curvature. This problem has not been solved yet, even for Finsler metrics of constant flag curvature.

According to E. Cartan's local classification theorem, any Riemannian metric $\alpha$ of constant sectional curvature $\mu$ is locally isometric to the following standard metric $\alpha_{\mu}$ on the unit ball $\mathrm{B}^{\mathrm{n}} \subset \mathrm{R}^{n}$ or the whole $\mathrm{R}^{n}$ for $\mu=-1,0,+1$ :

$$
\begin{array}{rlrl}
\alpha_{-1}(x, y) & =\frac{\sqrt{|y|^{2}-\left(|x|^{2}|y|^{2}-\langle x, y\rangle^{2}\right)}}{1-|x|^{2}}, & & y \in T_{x} \mathrm{~B}^{n} \cong \mathrm{R}^{n}, \\
\alpha_{0}(x, y) & =|y|, & y \in T_{x} \mathrm{R}^{n} \cong \mathrm{R}^{n}, \\
\alpha_{+1}(x, y) & =\frac{\sqrt{|y|^{2}+\left(|x|^{2}|y|^{2}-\langle x, y\rangle^{2}\right)}}{1+|x|^{2}} & & y \in T_{x} \mathrm{R}^{n} \cong \mathrm{R}^{n} .
\end{array}
$$

The simplest non-Riemannian Finsler metrics are those in the form $F=$ $\alpha+\beta$, where $\alpha$ is a Riemannian metric and $\beta$ is a 1 -form. They are called Randers metrics. Bao-Robles prove that if a Randers metric $F=\alpha+\beta$ has isotropic flag curvature $\mathbf{K}=\mathbf{K}(x)$, then there is a constant $c$ such that the covariant derivatives of $\beta$ with respect to $\alpha$ satisfy a system of PDEs 4 (i.e., equation (35) below with $c(x)=c$ ). Recently, Bao-Robles-Shen have classified Randers metrics of constant curvature via the navigation problem in Riemannian manifolds [5].

A Finsler metric is said to be locally projectively flat if at any point there is a local coordinate system in which the geodesics are straight lines as point sets. Why are we interested in these type of Finsler metrics? Riemannian metrics of constant curvature are locally projectively flat. The converse is true too according to Beltrami's theorem. Projectively flat Finsler metrics on a convex domain in $\mathrm{R}^{n}$ are regular solutions to Hilbert's Fourth Problem 18. It is known that every locally projectively flat Finsler metric is of scalar curvature. Locally projectively flat Finsler metrics with constant flag curvature have been solved at satisfactory level [7, 8, 9]-[1], 15-[17, 26, 27.

In Finsler geometry, there are several important non-Riemannian quantities: the distortion $\tau$, the mean Cartan torsion $\mathbf{I}$, the S-curvature $\mathbf{S}$ and the mean Landsberg curvature $\mathbf{J}$, etc. They all vanish for Riemannian metrics, hence they are said to be non-Riemannian. See Section 2 for more details about their definitions and geometric meanings.

All known Randers metrics $F=\alpha+\beta$ of scalar curvature (in dimension $n>2$ ) satisfy $\mathbf{S}=(n+1) c(x) F$ or $\mathbf{J}+c(x) F \mathbf{I}=0$, where $c(x)$ is a scalar curvature (see Theorem 1.3 below for projectively flat examples). In order to classify Finsler metrics of scalar curvature, we first investigate those with isotropic S-curvature. 
Theorem 1.1 Let $(M, F)$ be an n-dimensional Finsler manifold of scalar curvature with flag curvature $\mathbf{K}(x, y)$. Suppose that the S-curvature is isotropic,

$$
\mathbf{S}=(n+1) c(x) F(x, y),
$$

where $c(x)$ is a scalar function on $M$. Then there is a scalar function $\sigma(x)$ on $M$ such that

$$
\mathbf{K}=3 \frac{c_{x^{m}}(x) y^{m}}{F(x, y)}+\sigma(x) .
$$

In particular, $c(x)=c$ is a constant if and only if $\mathbf{K}=\mathbf{K}(x)$ is a scalar function on $M$.

In (5) and thereafter, the subscript $x^{m}$ in $c_{x^{m}}$ indicates partial differentiation with respect to $x^{m}$. In Theorem 1.1] we partially determine the flag curvature when the S-curvature is isotropic. This is a generalization of a theorem in [22] where the second author shows that the flag curvature is isotropic, $\mathbf{K}=\mathbf{K}(x)$ if (4) holds for $c(x)=$ constant. In this case, $\mathbf{K}=$ constant when $n \geq 3$ by the Schur theorem [3].

Theorem 1.2 Let $(M, F)$ be an n-dimensional Finsler manifold of scalar curvature. Suppose that $\mathbf{J} / \mathbf{I}$ is isotropic,

$$
\mathbf{J}+c(x) F \mathbf{I}=0,
$$

where $c=c(x)$ is a $C^{\infty}$ scalar function on $M$. Then the flag curvature $\mathbf{K}=$ $\mathbf{K}(x, y)$ and the distortion $\tau=\tau(x, y)$ satisfy

$$
\frac{n+1}{3} \mathbf{K}_{y^{k}}+\left(\mathbf{K}+c(x)^{2}-\frac{c_{x^{m}}(x) y^{m}}{F(x, y)}\right) \tau_{y^{k}}=0 .
$$

(a) If $c(x)=c$ is a constant, then there is a scalar function $\rho(x)$ on $M$ such that

$$
\mathbf{K}=-c^{2}+\rho(x) e^{-\frac{3 \tau(x, y)}{n+1}}, \quad y \in T_{x} M \backslash\{0\} .
$$

(b) Suppose that $F$ is non-Riemannian on any open subset of $M$. If $\mathbf{K}=\mathbf{K}(x)$ is a scalar function on $M$, then $c(x)=c$ is a constant, in which case $\mathbf{K}=-c^{2} \leq 0$.

From Theorem 1.2(a), it seems that (6) is weaker than (4) when $c(x)=$ constant. But for a non-Riemannian Randers metric $F=\alpha+\beta$, (6) implies (4) [12.

Finsler metrics with $\mathbf{J}=0$ are said to be weakly Landsbergian. Berwald metrics are weakly Landsbergian. According to Theorem [1.2 (a), for any weak Landsberg metric of scalar curvature, $\mathbf{K}=\rho(x) \exp \left(-\frac{3 \tau(x, y)}{n+1}\right)$. Here is an open problem: Is there any weak Landsberg metric of scalar curvature, which is nonBerwaldian? Further, one would like to know whether or not there are any 
non-Berwaldian Finsler metric of scalar curvature satisfying $\mathbf{J}+c F \mathbf{I}=0$ ? The only known examples are the family of (generalized) Funk metrics on the unit ball $\mathrm{B}^{n} \subset \mathrm{R}^{n}$ satisfying $\mathbf{J}+\frac{1}{2} F \mathbf{I}=0$ and $\mathbf{K}=-\frac{1}{4}$ (see Theorem [1.3 (A2) below).

Theorem 1.2 (a) in the case when $c=0$ is essentially proved in Matsumoto's book. See Proposition 26.2 in [19]. Matsumoto assumes that $F$ is a Landsberg metric, but what he actually needs in his proof is that $\mathbf{J}=0$. Since the notion of distortion has not been introduced in [19] by that time, Matsumoto's proposition is stated in a local coordinate system.

As we have mentioned early, locally projectively flat Finsler metrics are of scalar curvature. For a Randers metric $F=\alpha+\beta$, it is locally projectively flat and only if $\alpha$ is locally projectively flat (equivalently, of constant sectional curvature by Beltrami's theorem) and $\beta$ is closed [2] 23], in which case, (4) holds if and only if (6) holds [12. A natural problem is to determine $\beta$ such that $F=\alpha+\beta$ is locally projectively flat with $\mathbf{S}=(n+1) c(x) F$. Using Theorem 1.1. we find an explicit expression for $\beta$.

Theorem 1.3 Let $F=\alpha+\beta$ be a locally projectively flat Randers metric on an $n$-dimensional manifold $M$ and $\mu$ denote the constant sectional curvature of $\alpha$. Suppose that the $S$-curvature is isotropic, $\mathbf{S}=(n+1) c(x) F$. Then $F$ can be classified as follows.

(A) If $\mu+4 c(x)^{2} \equiv 0$, then $c(x)=$ constant and the flag curvature $\mathbf{K}=-c^{2}$.

(A1) if $c=0$, then $F$ is locally Minkowskian with flag curvature $\mathbf{K}=0$;

(A2) if $c \neq 0$, then after a normalization, $F$ is locally isometric to the following Randers metric on the unit ball $\mathrm{B}^{n} \subset \mathrm{R}^{n}$,

$$
F(x, y)=\frac{\sqrt{|y|^{2}-\left(|x|^{2}|y|^{2}-\langle x, y\rangle^{2}\right)} \pm\langle x, y\rangle}{1-|x|^{2}} \pm \frac{\langle a, y\rangle}{1+\langle a, x\rangle},
$$

where $a \in \mathrm{R}^{n}$ with $|a|<1$, and the flag curvature of $F$ is negative constant, $\mathbf{K}=-\frac{1}{4}$.

(B) If $\mu+4 c(x)^{2} \neq 0$, then $F$ is given by

$$
F(x, y)=\alpha(x, y)-\frac{2 c_{x^{k}}(x) y^{k}}{\mu+4 c(x)^{2}}
$$

and the flag curvature $\mathbf{K}$ of $F$ is given by

$$
\mathbf{K}=3\left\{\frac{c_{x^{k}}(x) y^{k}}{F(x, y)}+c(x)^{2}\right\}+\mu=\frac{3}{4}\left\{\mu+4 c(x)^{2}\right\} \frac{F(x,-y)}{F(x, y)}+\frac{\mu}{4} .
$$

(B1) when $\mu=-1, \alpha=\alpha_{-1}$ can be expressed in the form (1) on $\mathrm{B}^{n}$. In this case,

$$
c(x)=\frac{\lambda+\langle a, x\rangle}{2 \sqrt{(\lambda+\langle a, x\rangle)^{2} \pm\left(1-|x|^{2}\right)}},
$$

where $\lambda \in \mathrm{R}$ and $a \in \mathrm{R}^{n}$ with $|a|^{2}<\lambda^{2} \pm 1$. 
(B2) when $\mu=0, \alpha=\alpha_{0}$ can be expressed in the form (2) on $\mathrm{R}^{n}$. In this case,

$$
c(x)=\frac{ \pm 1}{2 \sqrt{k+2\langle a, x\rangle+|x|^{2}}},
$$

where $k>0$ and $a \in \mathrm{R}^{n}$ with $|a|^{2}<k$.

(B3) when $\mu=1, \alpha=\alpha_{+1}$ can be expressed in the form (3) on $\mathrm{R}^{n}$. In this case,

$$
c(x)=\frac{\epsilon+\langle a, x\rangle}{2 \sqrt{1+|x|^{2}-(\epsilon+\langle a, x\rangle)^{2}}},
$$

where $\epsilon \in \mathrm{R}$ and $a \in \mathrm{R}^{n}$ with $|\epsilon|^{2}+|a|^{2}<1$.

In a forthcoming paper, we are going to determine the local structures of projectively flat Finsler metrics with isotropic S-curvature.

Theorem 1.3 is a local classification theorem. If we assume that the manifold is compact without boundary, then the scalar function $c(x)$ takes much more special values.

Theorem 1.4 Let $F=\alpha+\beta$ be a locally projectively flat Randers metric on an $n$-dimensional compact manifold $M$ without boundary. Let $\mu$ denote the constant sectional curvature of $\alpha$. Suppose that $\mathbf{S}=(n+1) c(x) F$.

(a) If $\mu=-1$, then $F=\alpha$ is Riemannian.

(b) If $\mu=0$, then $F$ is locally Minkowskian.

(c) If $\mu=1$, then $c(x)=\frac{1}{2} f(x) / \sqrt{1-f(x)^{2}}$ and

$$
F(x, y)=\alpha(x, y)-\frac{f_{x^{k}}(x) y^{k}}{\sqrt{1-f(x)^{2}}},
$$

where $f(x)$ is an eigenfunction of the standard Laplacian corresponding to the eigenvalue $\lambda=n$ with $\max _{x \in M}|f|(x)<1$. Moreover, the flag curvature and the $S$-curvature of $F$ are given by

$$
\begin{gathered}
\mathbf{K}(x, y)=\frac{3}{4\left(1-f(x)^{2}\right)} \frac{F(x,-y)}{F(x, y)}+\frac{1}{4} . \\
\mathbf{S}(x, y)=\frac{(n+1) f(x)}{2 \sqrt{1-f(x)^{2}}} F(x, y) .
\end{gathered}
$$

In the case when $\mu=1,(M, \alpha)$ is isometric to the lense space $\mathrm{S}^{n} / \Gamma$. Let $F=\alpha+\beta$ be the Randers metric on $\mathrm{S}^{n}$ defined in (11) using some eigenfunction $f$ on $\mathrm{S}^{n}$. It can shown that $\delta:=\sqrt{|\nabla f|_{\alpha}^{2}(x)+f(x)^{2}}<1$ is a constant. By (12), we obtain the following bounds on the flag curvature of $F$.

$$
\frac{2-\delta}{2(1+\delta)} \leq \mathbf{K} \leq \frac{2+\delta}{2(1-\delta)}
$$

Assume that $(M, \alpha)=\mathrm{S}^{n}$ is the standard unit sphere. Since $F$ is pointwise projectively equivalent to $\alpha$, the geodesics of $F$ are great circles. One can easily see that the $F$-length of any great circle is equal to $2 \pi$. 


\section{Preliminaries}

In this section, we are going to give a brief description on several geometric quantities in Finsler geometry.

Let $F$ be a Finsler metric on an $n$-dimensional manifold $M$. The geodesics of $F$ are characterized by the following equations

$$
\ddot{c}^{i}(t)+2 G^{i}(c(t), \dot{c}(t))=0
$$

where $G^{i}=G^{i}(x, y)$ are given by

$$
G^{i}=\frac{1}{4} g^{i l}\left\{\left[F^{2}\right]_{x^{k} y^{l}} y^{k}-\left[F^{2}\right]_{x^{l}}\right\}
$$

where $g_{i j}(x, y)=\frac{1}{2}\left[F^{2}\right]_{y^{i} y^{j}}(x, y)$ and $\left(g^{i j}(x, y)\right):=\left(g_{i j}(x, y)\right)^{-1}$. When $F$ is Riemannian, i.e., $g_{i j}(x, y)=g_{i j}(x)$ depend only on $x \in M, G^{i}(x, y)=\frac{1}{2} \Gamma_{j k}^{i}(x) y^{j} y^{k}$ are quadratic in $y=\left.y^{i} \frac{\partial}{\partial x^{i}}\right|_{x}$. There are many non-Riemannian Finsler metrics with this property. Such Finsler metrics are called Berwald metrics.

Let

$$
\tau(x, y):=\ln \left[\frac{\sqrt{\operatorname{det}\left(g_{i j}(x, y)\right)}}{\operatorname{Vol}\left(\mathrm{B}^{n}(1)\right)} \cdot \operatorname{Vol}\left\{\left(y^{i}\right) \in \mathrm{R}^{n} \mid F\left(\left.y^{i} \frac{\partial}{\partial x^{i}}\right|_{x}\right)<1\right\}\right] .
$$

$\tau=\tau(x, y)$ is a scalar function on $T M \backslash\{0\}$, which is called the distortion [25].

Let

$$
I_{i}(x, y):=\frac{\partial \tau}{\partial y^{j}}(x, y)=\frac{1}{2} g^{j k}(x, y) \frac{\partial g_{j k}}{\partial y^{i}}(x, y) .
$$

The quantity $\mathbf{I}_{y}:=I_{i}(x, y) d x^{i}$ is called the mean Cartan torsion. According to Deicke's theorem, $F_{x}$ is Euclidean at $x \in M$ if and only if $\mathbf{I}_{y}=0$, or equivalently, $\tau=\tau(x)$ at $x \in M[3]$ [14].

Let

$$
\mathbf{S}(x, y):=\frac{d}{d t}[\tau(\sigma(t), \dot{\sigma}(t))]_{t=0},
$$

where $\sigma(t)$ is the geodesic with $\sigma(0)=x$ and $\dot{\sigma}(0)=y$. $\mathbf{S}$ is called the $S$ curvature 24 [25]. There are lots of Randers metrics of constant flag curvature satisfying $\mathbf{S}=(n+1) c F$ for some constant $c$ [6] [25] 28, 29]. $\mathbf{S}$ said to be isotropic if there is a scalar functions $c(x)$ on $M$ such that

$$
\mathbf{S}(x, y)=(n+1) c(x) F(x, y) .
$$

The horizontal covariant derivatives of $\mathbf{I}$ along geodesics give rise to the mean Landsberg curvature $\mathbf{J}_{y}:=J_{i}(x, y) d x^{i}$, where $J_{i}=J_{i}(x, y)$ are given by

$$
J_{i}:=y^{m} \frac{\partial I_{i}}{\partial x^{m}}-I_{m} \frac{\partial G^{m}}{\partial y^{i}}-2 G^{m} \frac{\partial I_{i}}{\partial y^{m}} .
$$

A Finsler metric $F$ is said to be weakly Landsbergian if $\mathbf{J}=0$. $\mathbf{J} / \mathbf{I}$ is regarded as the relative rate of change of $\mathbf{I}$ along geodesics. The generalized Funk metrics 
on the unit ball $\mathrm{B}^{n} \subset \mathrm{R}^{n}$ satisfy $\mathbf{J}+c F \mathbf{I}=0$ for some constant $c \neq 0$ [12] [26]. $\mathbf{J} / \mathbf{I}$ is said to be isotropic if there is a scalar function $c(x)$ on $M$ such that

$$
\mathbf{J}+c(x) F \mathbf{I}=0 .
$$

The Riemann curvature $\mathbf{K}_{y}=\left.K^{i}{ }_{k} d x^{k} \otimes \frac{\partial}{\partial x^{i}}\right|_{x}: T_{x} M \rightarrow T_{x} M$ is a family of linear maps on tangent spaces, defined by

$$
K_{k}^{i}=2 \frac{\partial G^{i}}{\partial x^{k}}-y^{j} \frac{\partial^{2} G^{i}}{\partial x^{j} \partial y^{k}}+2 G^{j} \frac{\partial^{2} G^{i}}{\partial y^{j} \partial y^{k}}-\frac{\partial G^{i}}{\partial y^{j}} \frac{\partial G^{j}}{\partial y^{k}} .
$$

For a flag $P=\operatorname{span}\{y, u\} \subset T_{x} M$ with flagpole $y$, the flag curvature $\mathbf{K}=$ $\mathbf{K}(P, y)$ is defined by

$$
\mathbf{K}(P, y):=\frac{\mathbf{g}_{y}\left(u, \mathbf{K}_{y}(u)\right)}{\mathbf{g}_{y}(y, y) \mathbf{g}_{y}(u, u)-\mathbf{g}_{y}(y, u)^{2}},
$$

where $\mathbf{g}_{y}=g_{i j}(x, y) d x^{i} \otimes d x^{j}$. When $F$ is Riemannian, $\mathbf{K}=\mathbf{K}(P)$ is independent of $y \in P$, which is just the sectional curvature of $P$ in Riemannian geometry. We say that a Finsler metric $F$ is of scalar curvature if for any $y \in T_{x} M$, the flag curvature $\mathbf{K}=\mathbf{K}(x, y)$ is a scalar function on the slit tangent bundle $T M \backslash\{0\}$. If $\mathbf{K}=$ constant, then $F$ is said to be of constant flag curvature.

It is easy to see that $F$ is locally projectively flat if and only if at any point there is a standard local coordinate system $\left(x^{i}, y^{i}\right)$ in $T M$ such that $G^{i}(x, y)=$ $P(x, y) y^{i}$. In this case, it follows from (13) that $K^{i}{ }_{k}=\Xi \delta_{k}^{i}+\tau_{k} y^{i}$. Thus $F$ is of scalar curvature.

Let $M$ be an $n$-dimensional manifold. Let $\pi^{*} T M$ denote the pull-back tangent bundle by $\pi: T M \backslash\{0\} \rightarrow M$ and $(x, y, v)$ denote the elements of $\pi^{*} T M$, where $y \in T_{x} M \backslash\{0\}$ and $v \in T_{x} M$. Let $\pi^{*} T^{*} M$ denote the horizontal cotangent bundle of $T M \backslash\{0\}$, consisting of $\pi^{*} \theta$, where $\theta \in T^{*} M$. There is a natural duality between $\pi^{*} T M$ and $\pi^{*} T^{*} M$. Let $\left\{\mathbf{e}_{i}:=\left(x, y, \frac{\partial}{\partial x^{i}}\right)\right\}$ be a natural local frame for $\pi^{*} T M$. Then $\left\{\omega^{i}:=\pi^{*} d x^{i}\right\}$ is the dual local coframe for $\pi^{*} T^{*} M$. $\pi^{*} T M$ has a canonical section, $Y:=(x, y, y)=y^{i} \mathbf{e}_{i}$, where $y=y^{i} \frac{\partial}{\partial x^{i}}$.

Given a Finsler metric $F$ on $M$. It defines the Riemannian metric tensor $\mathbf{g}=g_{i j} \omega^{i} \otimes \omega^{j}$ and the Cartan torsion $\mathbf{C}=C_{i j k} \omega^{i} \otimes \omega^{j} \otimes \omega^{k}$ on $\pi^{*} T M$, where $g_{i j}=\frac{1}{2}\left[F^{2}\right]_{y^{i} y^{j}}$ and $C_{i j k}=\frac{1}{4}\left[F^{2}\right]_{y^{i} y^{j} y^{k}}$. The Chern connection is a linear connection on $\pi^{*} T M$, which are characterized by

$$
\begin{gathered}
d \omega^{i}=\omega^{j} \wedge \omega_{j}^{i} \\
d g_{i j}=g_{i k} \omega_{j}^{k}+g_{k j} \omega_{i}^{k}+2 C_{i j k}\left\{d y^{k}+y^{j} \omega_{j}^{k}\right\} .
\end{gathered}
$$

See [3] 13. Let

$$
\omega^{n+k}:=d y^{k}+y^{j} \omega_{j}^{k}
$$


We obtain a local coframe $\left\{\omega^{i}, \omega^{n+i}\right\}$ for $T^{*}(T M \backslash\{0\})$. Let

$$
\Omega^{i}:=d \omega^{n+i}-\omega^{n+j} \wedge \omega_{j}^{i} .
$$

We can express $\Omega^{i}$ in the following form

$$
\Omega^{i}=\frac{1}{2} K_{k l}^{i} \omega^{k} \wedge \omega^{l}-L_{k l}^{i} \omega^{k} \wedge \omega^{n+l},
$$

where $K_{k l}^{i}+K_{l k}^{i}=0$. Let

$$
K_{k}^{i}:=K_{k l}^{i} y^{l} .
$$

We obtain the Riemann curvature $\mathbf{K}=K^{i}{ }_{k} \omega^{k} \otimes \omega^{l}$ and $\mathbf{L}=L_{k l}^{i} \omega^{k} \otimes \omega^{l} \otimes \mathbf{e}_{i}$. In a standard local coordinate system $\left(x^{i}, y^{i}\right), K^{i}{ }_{k}$ are given by (13). The Riemann curvature is introduced by Riemann in 1854 for Riemannian metrics and extended to Finsler metrics by L. Berwald in 1926 [7] 8].

With the Chern connection, we define covariant derivatives of quantities on $T M \backslash\{0\}$ in the usual way. For example, for a scalar function $f$, we define $f_{\mid i}$ and $f_{\cdot i}$ by

$$
d f=f_{\mid i} \omega^{i}+f_{\cdot i} \omega^{n+i},
$$

for the mean Cartan torsion $\mathbf{I}=I_{i} \omega^{i}$, define $I_{i \mid j}$ and $I_{i \cdot j}$ by

$$
d I_{i}-I_{k} \omega_{i}^{k}=I_{i \mid j} \omega^{j}+I_{i \cdot j} \omega^{n+j} .
$$

For a tensor $\mathbf{T}=T_{i \cdots k} \omega^{i} \otimes \cdots \otimes \omega^{k}$,

$$
T_{i \cdots k \cdot m}=\frac{\partial T_{i \cdots k}}{\partial y^{m}} .
$$

Without much difficulty, one can show that

$$
K_{k l}^{i}=\frac{1}{3}\left\{K_{k \cdot l}^{i}-K_{l \cdot k}^{i}\right\}
$$

and

$$
I_{i}=\tau_{\cdot i}, \quad \mathbf{S}:=\tau_{\mid m} y^{m}, \quad J_{i}=I_{i \mid m} y^{m} .
$$

Moreover, $L_{i j k}:=g_{i m} L_{k l}^{m}=C_{i j k \mid m} y^{m}$. See [25]. The following equations are proved in [21] 23].

$$
\begin{aligned}
L_{i j k \mid m} y^{m}+C_{i j m} K_{k}^{m}= & -\frac{1}{3} g_{i m} K_{k \cdot j}^{m}-\frac{1}{3} g_{j m} K_{k \cdot i}^{m} \\
& -\frac{1}{6} g_{i m} K_{j \cdot k}^{m}-\frac{1}{6} g_{j m} K_{i \cdot k}^{m} .
\end{aligned}
$$

Contracting (15) with $g^{i j}$ gives

$$
J_{k \mid m} y^{m}+I_{m} K_{k}^{m}=-\frac{1}{3}\left\{2 K_{k \cdot m}^{m}+K_{m \cdot k}^{m}\right\} .
$$


As a scalar function on $T M \backslash\{0\}$, the distortion satisfies the following Ricci identities

$$
\begin{aligned}
\tau_{|k| l} & =\tau_{|l| k}+\tau_{\cdot m} K_{k l}^{m}, \\
\tau_{\mid k \cdot l} & =\tau_{\cdot l \mid k}-\tau_{\cdot m} L_{k l}^{m} .
\end{aligned}
$$

Contracting (18) with $y^{k}$ yields

$$
\mathbf{S}_{. l}=\tau_{\mid l}+J_{l} .
$$

It follows from (19) that

$$
\mathbf{S}_{\cdot k \mid l}=\tau_{|k| l}+J_{k \mid l} .
$$

Using (14), (17), and (20), we obtain

$$
\begin{aligned}
\mathbf{S}_{\cdot k \mid l} y^{l}-\mathbf{S}_{\mid k} & =\left(\mathbf{S}_{\cdot k \mid l}-\mathbf{S}_{\cdot l \mid k}\right) y^{l} \\
& =\left(\tau_{|k| l}-\tau_{|l| k}\right) y^{l}+\left(J_{k \mid l}-J_{l \mid k}\right) y^{l} \\
& =\tau_{\cdot m} K_{k l}^{m} y^{l}-I_{m} K_{k}^{m}-\frac{1}{3}\left\{2 K_{k \cdot m}^{m}+K_{m \cdot k}^{m}\right\} \\
& =-\frac{1}{3}\left\{2 K_{k \cdot m}^{m}+K_{m \cdot k}^{m}\right\} .
\end{aligned}
$$

We obtain

$$
\mathbf{S}_{\cdot k \mid m} y^{m}-\mathbf{S}_{\mid k}=-\frac{1}{3}\left\{2 K_{k \cdot m}^{m}+K_{m \cdot k}^{m}\right\} .
$$

Equation (21) is established in 22.

Now we assume that $F$ is of scalar curvature with flag curvature $\mathbf{K}=\mathbf{K}(x, y)$. This is equivalent to the following identity:

$$
K^{i}{ }_{k}=\mathbf{K} F^{2} h_{k}^{i},
$$

where $h_{k}^{i}:=g^{i j} h_{j k}$ and $h_{j k}:=g_{j k}-F^{-2} g_{j s} y^{s} g_{k t} y^{t}$. By (15), (16) and (22), we obtain

$$
L_{i j k \mid m} y^{m}=-\frac{1}{3} F^{2}\left\{\mathbf{K}_{\cdot i} h_{j k}+\mathbf{K}_{\cdot j} h_{i k}+\mathbf{K}_{\cdot k} h_{i j}+3 \mathbf{K} C_{i j k}\right\}
$$

and

$$
J_{k \mid m} y^{m}=-\frac{1}{3} F^{2}\left\{(n+1) \mathbf{K}_{\cdot k}+3 \mathbf{K} I_{k}\right\} .
$$

\section{Proofs of Theorems 1.1 and 1.2}

In this section, we are going to prove the first two theorems.

Proof of Theorem 1.1] Plugging (22) into (21), we obtain

$$
\mathbf{S}_{\cdot k \mid l} y^{l}-\mathbf{S}_{\mid k}=-\frac{n+1}{3} \mathbf{K}_{\cdot k} F^{2} .
$$


Plugging (4) into (24) yields

$$
c_{\mid l}(x) y^{l} F_{\cdot k}-c_{\mid k}(x) F=-\frac{1}{3} \mathbf{K}_{\cdot k} F^{2} .
$$

It follows from (25) that

$$
\left[\frac{1}{3} \mathbf{K}-\frac{c_{\mid m}(x) y^{m}}{F(x, y)}\right]_{y^{k}}=0 .
$$

Thus

$$
\sigma:=\mathbf{K}-\frac{3 c_{\mid m} y^{m}}{F}
$$

is a scalar function on $M$. This proves the theorem.

Q.E.D.

Proof of Theorem 1.2 By assumption, $J_{k}=-c F I_{k}$ and $J_{k}=I_{k \mid m} y^{m}$ we obtain

$$
J_{k \mid m} y^{m}=-c_{\mid m} y^{m} F I_{k}-c F I_{k \mid m} y^{m}=-c_{\mid m} y^{m} F I_{k}+c^{2} F^{2} I_{k} .
$$

It follows from (23) that

$$
\frac{n+1}{3} \mathbf{K}_{\cdot k}+\left(\mathbf{K}+c^{2}-\frac{c_{x^{m}} y^{m}}{F}\right) I_{k}=0
$$

By (14), $I_{k}=\tau_{\cdot k}$. We obtain (7).

(a) Suppose that $c_{x^{m}}(x)=0$ at some point $x \in M$. Then equation (7) simplifies to

$$
\frac{n+1}{3} \mathbf{K}_{y^{k}}+\left(\mathbf{K}+c^{2}\right) \tau_{y^{k}}=0
$$

This implies that

$$
\left[\left(\mathbf{K}+c^{2}\right)^{\frac{n+1}{3}} e^{\tau}\right]_{y^{k}}=\left(\mathbf{K}+c^{2}\right)^{\frac{n-2}{3}} e^{\tau}\left\{\frac{n+1}{3} \mathbf{K}_{y^{k}}+\left(\mathbf{K}+c^{2}\right) \tau_{y^{k}}\right\}=0 .
$$

Thus the function $\left(\mathbf{K}+c^{2}\right)^{\frac{n+1}{3}} e^{\tau}$ is independent of $y \in T_{x} M$. There is a number $\rho(x)$ such that

$$
\mathbf{K}=-c(x)^{2}+\rho(x) e^{-\frac{3 \tau(x, y)}{n+1}} .
$$

When $c(x)=c$ is a constant, we obtain (8) from (27). Note that $\rho(x)$ is not necessarily a constant.

(b) Suppose that $\mathbf{K}=\mathbf{K}(x)$ is a scalar function on $M$. Then (17) simplifies to

$$
\left(\mathbf{K}+c^{2}-\frac{c_{x^{m}} y^{m}}{F}\right) \tau_{y^{k}}=0 .
$$

We claim that $c(x)=c$ is a constant. If this is false, then there is an open subset $\mathcal{U}$ such that $d c(x) \neq 0$ for any $x \in \mathcal{U}$. Clearly, at any $x \in \mathcal{U}, \mathbf{K}(x) \neq$ $-c(x)^{2}+c_{x^{m}}(x) y^{m} / F(x, y)$ for almost all $y \in T_{x} M$. By (28), $\tau_{\cdot k}=I_{k}=0$. Thus $F$ is Riemannian on $\mathcal{U}$ by Deicke's theorem (cf. 14] [3]). This contradicts 
our assumption in the theorem. This proves the claim. By (27) and (28), we obtain

$$
\rho(x) \tau_{y^{k}}=0 .
$$

We claim that $\rho(x) \equiv 0$. If this is false, then there is an open subset $\mathcal{U}$ such that $\rho(x) \neq 0$ for any $x \in \mathcal{U}$. By (29), we obtain that $\tau_{y^{k}}=I_{k}=0$ on $\mathcal{U}$. Thus $F$ is Riemannian on $\mathcal{U}$. This again contradicts the assumption in the theorem. Therefore $\rho(x) \equiv 0$. We conclude that $\mathbf{K}=-c^{2}$ by (27).

Q.E.D.

According to 12, for any Randers metric $F=\alpha+\beta$, (6) holds if and only if (4) holds and $\beta$ is closed. For a general Finsler metric, (6) does not imply (4). Now we combine two conditions (44) and (6) and prove the following

Theorem 3.1 Let $(M, F)$ be an n-dimensional Finsler manifold of scalar curvature. Suppose that the S-curvature and the mean Landsberg curvature satisfy

$$
\mathbf{S}=(n+1) c(x) F, \quad \mathbf{J}+c(x) F \mathbf{I}=0,
$$

where $c=c(x)$ is a scalar function on $M$. Then the flag curvature is given by

$$
\mathbf{K}=3 \frac{c_{x^{m}}(x) y^{m}}{F(x, y)}+\sigma(x)=-\frac{3 c(x)^{2}+\sigma(x)}{2}+\nu(x) e^{\frac{-2 \tau(x, y)}{n+1}},
$$

where $\sigma(x)$ and $\nu(x)$ are scalar functions on $M$.

(a) Suppose that $F$ is not Riemannian on any open subset in $M$. If $c(x)=c$ is a constant, then $\mathbf{K}=-c^{2}, \sigma(x)=-c^{2}$ and $\nu(x)=0$.

(b) If $c(x) \neq$ constant, then the distortion is given by

$$
\tau=\ln \left\{\frac{2 \nu(x) F(x, y)}{6 c_{x^{m}}(x) y^{m}+3\left[\sigma(x)+c(x)^{2}\right] F(x, y)}\right\}^{\frac{2}{n+1}} .
$$

Proof: By the above argument, $\mathbf{K}$ is given by (5) and it satisfies (7). It follows from (5) that

$$
\frac{c_{x^{m}}(x) y^{m}}{F(x, y)}=\frac{1}{3}(\mathbf{K}-\sigma(x)) .
$$

Plugging it into (7) yields

$$
\frac{n+1}{3} \mathbf{K}_{y^{k}}+\left(\frac{2}{3} \mathbf{K}+c(x)^{2}+\frac{1}{3} \sigma(x)\right) \tau_{y^{k}}=0 .
$$

We obtain

$$
\left[\left(2 \mathbf{K}+3 c(x)^{2}+\sigma(x)\right)^{\frac{n+1}{2}} e^{\tau}\right]_{y^{k}}=0 .
$$

Thus there is a scalar function $\nu(x)$ on $M$ such that

$$
\mathbf{K}=-\frac{3 c(x)^{2}+\sigma(x)}{2}+\nu(x) e^{-\frac{2 \tau(x, y)}{n+1}} .
$$


Comparing (33) with (5), we obtain

$$
\frac{c_{x^{m}}(x) y^{m}}{F(x, y)}=-\frac{c(x)^{2}+\sigma(x)}{2}+\frac{\nu(x)}{3} e^{-\frac{2 \tau(x, y)}{n+1}} .
$$

(a) Suppose that $c(x)=c$ is a constant. We claim that $\nu(x)=0$. If it false, then $\mathcal{U}:=\{x \in M, \nu(x) \neq 0\} \neq \emptyset$. From (34), one can see that $\tau=\tau(x)$ is a scalar function on $\mathcal{U}$, hence $F$ is Riemannian on $\mathcal{U}$ by Deicke's theorem [14] [3]. This contradicts the assumption in (a).

Now (34) is reduced to that $\sigma(x)=c(x)^{2}$ and (33) is reduced to that $\mathbf{K}=$ $-c^{2}$.

(b) If $c(x) \neq$ contant, then $\nu(x) \neq 0$ by (34). In this case, we can solve (34) for $\tau$ and obtain (32).

Q.E.D.

It follows from Theorem 3.1 that if a Finsler metric of scalar curvature satisfies that $\mathbf{S}=(n+1) c F$ and $\mathbf{J}+c F \mathbf{I}=0$ for some constant $c$, then the flag curvature is given by $\mathbf{K}=-c^{2}$. One would like to know whether or not there are non-Riemannian, non-locally Minkowskian Finsler metrics with these properties. If a Randers metric has these properties, then it is, up to a scaling, locally isometric to the generalized Funk metric on the unit ball $\mathrm{B}^{n} \subset \mathrm{R}^{n}[12$. In dimension two, any Finsler metric with $\mathbf{S}=0, \mathbf{J}=0$ and $\mathbf{K}=0$ is locally Minkowskian.

Example 3.2 For an arbitrary number $\epsilon$ with $0<\epsilon \leq 1$, define

$$
\begin{aligned}
& \alpha:=\frac{\sqrt{\left(1-\epsilon^{2}\right)(x u+y v)^{2}+\epsilon\left(u^{2}+v^{2}\right)\left(1+\epsilon\left(x^{2}+y^{2}\right)\right)}}{1+\epsilon\left(x^{2}+y^{2}\right)} \\
& \beta:=\frac{\sqrt{1-\epsilon^{2}}(x u+y v)}{1+\epsilon\left(x^{2}+y^{2}\right)} .
\end{aligned}
$$

We have

$$
\|\beta\|_{\alpha}=\sqrt{1-\epsilon^{2}} \sqrt{\frac{x^{2}+y^{2}}{\epsilon+x^{2}+y^{2}}}<1 .
$$

Thus $F:=\alpha+\beta$ is a Randers metric on $\mathrm{R}^{2}$. In [12, we have verified that

$$
\mathbf{S}=3 c F, \quad \mathbf{J}_{y}+c F \mathbf{I}_{y}=0
$$

where

$$
c=\frac{\sqrt{1-\epsilon^{2}}}{2\left(\epsilon+x^{2}+y^{2}\right)},
$$

and obtained a formula for the Gauss curvature

$$
\begin{aligned}
\mathbf{K}= & \frac{-3 \sqrt{1-\epsilon^{2}}(x u+y v) /\left(1+\epsilon\left(x^{2}+y^{2}\right)\right)}{\sqrt{\left(1-\epsilon^{2}\right)(x u+y v)^{2}+\epsilon\left(u^{2}+v^{2}\right)\left(1+\epsilon\left(x^{2}+y^{2}\right)\right)}+\sqrt{1-\epsilon^{2}}(x u+y v)} \\
& +\frac{7\left(1-\epsilon^{2}\right)+8 \epsilon\left(\epsilon+x^{2}+y^{2}\right)}{4\left(\epsilon+x^{2}+y^{2}\right)^{2}} .
\end{aligned}
$$


Here we are going to compute $\sigma$ and $\nu$ in Theorem 3.1 By a direct computation we can express the function $\sigma:=\mathbf{K}-\frac{3\left(c_{x} u+c_{y} v\right)}{F}$ in (31) by

$$
\sigma=\frac{7\left(1-\epsilon^{2}\right)}{4\left(\epsilon+x^{2}+y^{2}\right)^{2}}+\frac{2 \epsilon}{\epsilon+x^{2}+y^{2}} .
$$

That is, the Gauss curvature is given by

$$
\begin{aligned}
\mathbf{K} & =3 \frac{c_{x} u+c_{y} v}{F}+\sigma \\
& =-\frac{3 \sqrt{1-\epsilon^{2}}(x u+y v)}{\left(\epsilon+x^{2}+y^{2}\right)^{2} F}+\frac{7\left(1-\epsilon^{2}\right)}{4\left(\epsilon+x^{2}+y^{2}\right)^{2}}+\frac{2 \epsilon}{\epsilon+x^{2}+y^{2}} .
\end{aligned}
$$

For any Randers metric $F=\alpha+\beta$, the distortion is given by

$$
\tau=\ln \left[\frac{F}{\alpha} \cdot \frac{1}{1-\|\beta\|_{\alpha}^{2}}\right]^{\frac{n+1}{2}} .
$$

A direct computation yields

$$
1-\|\beta\|_{\alpha}^{2}=\frac{\epsilon\left(1+\epsilon\left(x^{2}+y^{2}\right)\right)}{\epsilon+x^{2}+y^{2}} .
$$

Then the function $\nu:=\left(\mathbf{K}+\frac{3 c^{2}+\sigma}{2}\right) e^{\frac{2 \tau}{n+1}}$ in (31) is given by

$$
\nu=\frac{3}{\epsilon\left(\epsilon+x^{2}+y^{2}\right)} \text {. }
$$

That is, the Gauss curvature can also be given by

$$
\begin{aligned}
\mathbf{K} & =-\frac{3 c^{2}+\sigma}{2}+\nu e^{-\frac{2 \tau}{n+1}} \\
& =-\frac{5-\epsilon^{2}+4 \epsilon\left(x^{2}+y^{2}\right)}{2\left(\epsilon+x^{2}+y^{2}\right)^{2}}+\frac{3\left(1+\epsilon\left(x^{2}+y^{2}\right)\right) \alpha}{\left(\epsilon+x^{2}+y^{2}\right)^{2} F} .
\end{aligned}
$$

\section{Proof of Theorem 1.3}

Let $F=\alpha+\beta$ be a Randers metric on an $n$-dimensional manifold $M$, where $\alpha=\sqrt{a_{i j}(x) y^{i} y^{j}}$ and $\beta=b_{i}(x) y^{i}$. Throughout this paper, we always assume that $F$ is positive definite or $\|\beta\|_{\alpha}(x):=\sqrt{a^{i j}(x) b_{i}(x) b_{j}(x)}<1$ for any $x \in M$. Define $b_{i \mid j}$ by

$$
b_{i \mid j} \theta^{j}:=d b_{i}-b_{j} \theta_{i}{ }^{j},
$$

where $\theta^{i}:=d x^{i}$ and $\theta_{i}{ }^{j}:=\gamma_{j k}^{i} d x^{k}$ denote the Levi-Civita connection forms of $\alpha$. Let

$$
r_{i j}:=\frac{1}{2}\left(b_{i \mid j}+b_{j \mid i}\right), \quad s_{i j}:=\frac{1}{2}\left(b_{i \mid j}-b_{j \mid i}\right),
$$




$$
s^{i}{ }_{j}:=a^{i h} s_{h j}, \quad s_{j}:=b_{i} s_{j}^{i}, \quad e_{i j}:=r_{i j}+b_{i} s_{j}+b_{j} s_{i} .
$$

Let

$$
\rho(x):=\ln \sqrt{1-\|\beta\|_{\alpha}^{2}(x)}
$$

and $d \rho=\rho_{i} d x^{i}$. According to 24, the S-curvature of $F=\alpha+\beta$ is given by

$$
\mathbf{S}=(n+1)\left\{\frac{e_{00}}{2 F}-\left(s_{0}+\rho_{0}\right)\right\},
$$

where $e_{00}:=e_{i j} y^{i} y^{j}$ and $s_{0}:=s_{i} y^{i}$ and $\rho_{0}:=\rho_{p} y^{p}$. According to Lemma 3.1 in [12], $\mathbf{S}=(n+1) c(x) F$ is equivalent to that

$$
e_{i j}=2 c(x)\left(a_{i j}-b_{i} b_{j}\right) .
$$

Assume that $\alpha$ is of constant sectional curvature and $\beta$ is closed (hence $s_{i j}=0$ and $\left.s_{i}=0\right)$. Let

$$
\Phi:=b_{i \mid j} y^{i} y^{j}, \quad \Psi:=b_{i|j| k} y^{i} y^{j} y^{k} .
$$

By (8.56) in [24, we have

$$
\mathbf{K} F^{2}=\mu \alpha^{2}+3\left[\frac{\Phi}{2 F}\right]^{2}-\frac{\Psi}{2 F} .
$$

Further we assume that $\mathbf{S}=(n+1) c(x) F$. Since $s_{i j}=0, e_{i j}=r_{i j}=b_{i \mid j}$ and (35) simplifies to

$$
b_{i \mid j}=2 c\left(a_{i j}-b_{i} b_{j}\right) .
$$

We obtain

$$
\begin{aligned}
& \Phi=2 c\left(\alpha^{2}-\beta^{2}\right) \\
& \Psi=2 c_{x^{k}} y^{k}\left(\alpha^{2}-\beta^{2}\right)-8 c^{2}\left(\alpha^{2}-\beta^{2}\right) \beta .
\end{aligned}
$$

Now we are ready to prove Theorem 1.3 Let $F=\alpha+\beta$ be a Randers metric in Theorem 1.3. Since $F$ is locally projectively flat, $\alpha$ is locally projectively flat and $\beta$ is closed 23]. By the Beltrami theorem, we know that $\alpha$ is of constant sectional curvature $\mu$. Our main task is to determine $\beta$.

By Theorem 1.1 we know that the flag curvature is in the following form

$$
\mathbf{K}=\frac{3 c_{x^{k}}(x) y^{k}}{F(x, y)}+\sigma(x)
$$

where $\sigma(x)$ is a scalar function on $M$. It follows from (36) and (37) that

$$
3 c_{x^{k}} y^{k} F+\sigma F^{2}=\mathbf{K} F^{2}=\mu \alpha^{2}+3\left[\frac{\Phi}{2 F}\right]^{2}-\frac{\Psi}{2 F} .
$$

Using the above formulas for $\Phi$ and $\Psi$, we obtain

$$
2\left\{2 c_{x^{k}} y^{k}+\left(\sigma+c^{2}\right) \beta\right\} \alpha+\left\{2 c_{x^{k}} y^{k}+\left(\sigma+c^{2}\right) \beta\right\} \beta+\left\{\sigma-3 c^{2}-\mu\right\} \alpha^{2}=0 .
$$


This gives

$$
\begin{aligned}
& 2 c_{x^{k}} y^{k}+\left(\sigma+c^{2}\right) \beta=0, \\
& \sigma-3 c^{2}-\mu=0 .
\end{aligned}
$$

Plugging (39) into (37) and (38) yields

$$
\begin{gathered}
\mathbf{K}=3\left\{\frac{c_{x^{k}}(x) y^{k}}{F(x, y)}+c(x)^{2}\right\}+\mu . \\
2 c_{x^{k}} y^{k}+\left(\mu+4 c^{2}\right) \beta=0 .
\end{gathered}
$$

Now we are ready to determine $\beta$ and $c$.

Case 1: Suppose that $\mu+4 c(x)^{2} \equiv 0$. Then $c(x)=c$ is a constant. It follows from (40) that

$$
\mathbf{K}=3 c^{2}+\mu=-c^{2} .
$$

Then Theorem 1.3 (A) follows from the classification theorem for projectively flat Randers of constant curvature [25].

Case 2: Suppose that $\mu+4 c(x)^{2} \neq 0$ on an open subset $\mathcal{U} \subset M$. It follows from (41) that

$$
\beta=-\frac{2 c_{x^{k}}(x) y^{k}}{\mu+4 c(x)^{2}}
$$

Note that $\beta$ is exact. Let $c_{i} d x^{i}:=d c$ and $c_{i \mid j} d x^{j}:=d c_{i}-c_{k} \bar{\Gamma}_{i j}^{k} d x^{j}$ denote the covariant derivative of $d c$ with respect to $\alpha$, were $\bar{\Gamma}_{i j}^{k}$ denote the Christoffel symbols of $\alpha$. We have

$$
c_{i}=c_{x^{i}}(x), \quad c_{i \mid j}=c_{x^{i} x^{j}}(x)-c_{x^{k}}(x) \bar{\Gamma}_{i j}^{k}(x) .
$$

Similarly, we can define $b_{i \mid j}$ and $b_{i|j| k}$. Since $\beta$ is closed, $b_{i \mid j}=b_{j \mid i}$. In this case, $\mathbf{S}=(n+1) c(x) F$ is equivalent to

$$
b_{i \mid j}=2 c\left(a_{i j}-b_{i} b_{j}\right) .
$$

From (42), we have

$$
b_{i}=-\frac{2 c_{i}}{\mu+4 c^{2}} .
$$

Plugging (44) into (43) yields

$$
c_{i \mid j}=-c\left(\mu+4 c^{2}\right) a_{i j}+\frac{12 c c_{i} c_{j}}{\mu+4 c^{2}} .
$$

Next we are going to solve (45) for $c(x)$ in three cases when $\mu=-1,0,1$. 
(B1): $\mu=-1$. We assume that $\alpha=\alpha_{-1}=\sqrt{a_{i j}(x) y^{i} y^{j}}$ which is expressed in the form (1). We have

$$
a_{i j}=\frac{\delta_{i j}}{1-|x|^{2}}+\frac{x^{i} x^{j}}{\left(1-|x|^{2}\right)^{2}} .
$$

The Christoffel symbols of $\alpha$ are given by

$$
\bar{\Gamma}_{i j}^{k}=\frac{x^{i} \delta_{j}^{k}+x^{j} \delta_{i}^{k}}{1-|x|^{2}}
$$

Equation (45) becomes

$$
c_{x^{i} x^{j}}-\frac{x^{i} c_{x^{j}}+x^{j} c_{x^{i}}}{1-|x|^{2}}=-c\left(-1+4 c^{2}\right)\left\{\frac{\delta_{i j}}{1-|x|^{2}}+\frac{x^{i} x^{j}}{\left(1-|x|^{2}\right)^{2}}\right\}+\frac{12 c c_{x^{i}} c_{x^{j}}}{-1+4 c^{2}} .
$$

Let

$$
f:=\frac{2 c \sqrt{1-|x|^{2}}}{\sqrt{\mp\left(-1+4 c^{2}\right)}}
$$

where the sign depends on the value of $c$ such that $\mp\left(-1+4 c^{2}\right)>0$. Equation (46) simplifies to

$$
f_{x^{i} x^{j}}=0 .
$$

We obtain that $f=\langle a, x\rangle+\lambda$, where $\lambda \in \mathrm{R}$ and $a \in \mathrm{R}^{n}$. Then we obtain

$$
c=\frac{\lambda+\langle a, x\rangle}{2 \sqrt{(\lambda+\langle a, x\rangle)^{2} \pm\left(1-|x|^{2}\right)}}
$$

Plugging (47) into (42) yields

$$
\beta=\frac{(\lambda+\langle a, x\rangle)\langle x, y\rangle+\left(1-|x|^{2}\right)\langle a, y\rangle}{\left(1-|x|^{2}\right) \sqrt{(\lambda+\langle a, x\rangle)^{2} \pm\left(1-|x|^{2}\right)}}
$$

and

$$
F=\frac{\sqrt{|y|^{2}-\left(|x|^{2}|y|^{2}-\langle x, y\rangle^{2}\right)}}{1-|x|^{2}}+\frac{(\lambda+\langle a, x\rangle)\langle x, y\rangle+\left(1-|x|^{2}\right)\langle a, y\rangle}{\left(1-|x|^{2}\right) \sqrt{(\lambda+\langle a, x\rangle)^{2} \pm\left(1-|x|^{2}\right)}} .
$$

By a direct computation ,

$$
1-\|\beta\|_{\alpha}^{2}=\frac{\left(1-|x|^{2}\right)\left\{ \pm 1-\left(|a|^{2}-\lambda^{2}\right)\right\}}{(\lambda+\langle a, x\rangle)^{2} \pm\left(1-|x|^{2}\right)} .
$$

Clearly, $F=\alpha+\beta$ is a Randers metric on an open subset of $\mathrm{B}^{n}$ if and only if $|a|^{2}-\lambda^{2}< \pm 1$. In this case, $(\lambda+\langle a, x\rangle)^{2} \pm\left(1-|x|^{2}\right)>0$ for any $x \in \mathrm{B}^{n}$. Thus $F$ can be extended to the whole $\mathrm{B}^{n}$. By (40), (47) and (48), we obtain

$$
\mathbf{K}=-\frac{3}{4} \frac{ \pm\left(1-|x|^{2}\right)}{(\lambda+\langle a, x\rangle)^{2} \pm\left(1-|x|^{2}\right)} \cdot \frac{F(x,-y)}{F(x, y)}-\frac{1}{4} .
$$


(B2) $\mu=0$. We assume that $\alpha=\alpha_{0}=\sqrt{\delta_{i j} y^{i} y^{j}}$ which is expressed in the form (2). Equation (45) becomes

$$
c_{x^{i} x^{j}}=-4 c^{3} \delta_{i j}+\frac{3 c_{x^{i}} c_{x^{j}}}{c} .
$$

Let $\mathcal{U}:=\left\{x \in \mathrm{R}^{n} \mid c(x) \neq 0\right\}$ and let

$$
f=\frac{1}{c^{2}} .
$$

Equation (49) simplifies to

$$
f_{x^{i} x^{j}}=8 \delta_{i j}
$$

We obtain

$$
f=4\left(k+2\langle a, x\rangle+|x|^{2}\right),
$$

where $k \in \mathrm{R}$ and $a \in \mathrm{R}^{n}$ such that $f(x)>0$ for $x \in \mathcal{U}$. Then $c= \pm 1 / \sqrt{f}$ is given by

$$
c=\frac{ \pm 1}{2 \sqrt{k+2\langle a, x\rangle+|x|^{2}}} .
$$

Plugging (51) into (42) yields

$$
\beta= \pm \frac{\langle a, y\rangle+\langle x, y\rangle}{\sqrt{k+2\langle a, x\rangle+|x|^{2}}},
$$

and

$$
F=|y| \pm \frac{\langle a, y\rangle+\langle x, y\rangle}{\sqrt{k+2\langle a, x\rangle+|x|^{2}}}
$$

Note that

$$
1-\|\beta\|_{\alpha}^{2}=\frac{k-|a|^{2}}{k+2\langle a, x\rangle+|x|^{2}} .
$$

Clearly, $F=\alpha+\beta$ is a Randers metric on an open subset of $\mathrm{R}^{n}$ if and only if $|a|^{2}<k$. In this case,

$$
k+2\langle a, x\rangle+|x|^{2} \geq k-|a|^{2}+(|a|-|x|)^{2}>0, \quad \forall x \in \mathrm{R}^{n} .
$$

Thus $F$ can be extended to the whole $\mathrm{R}^{n}$. By (40), (51) and (52), we obtain

$$
\mathbf{K}=\frac{3}{4\left(k+2\langle a, x\rangle+|x|^{2}\right)} \cdot \frac{F(x,-y)}{F(x, y)}>0 .
$$

(B3) $\mu=+1$. We assume that $\alpha=\alpha_{+1}=\sqrt{a_{i j}(x) y^{i} y^{j}}$ which is expressed in the form (3). We have

$$
a_{i j}=\frac{\delta_{i j}}{1+|x|^{2}}-\frac{x^{i} x^{j}}{\left(1+|x|^{2}\right)^{2}}
$$


The Christoffel symbols of $\alpha$ are given by

$$
\bar{\Gamma}_{i j}^{k}=-\frac{x^{i} \delta_{j}^{k}+x^{j} \delta_{i}^{k}}{1+|x|^{2}} .
$$

Equation (45) becomes

$$
c_{x^{i} x^{j}}+\frac{x^{i} c_{x^{j}}+x^{j} c_{x^{i}}}{1+|x|^{2}}=-c\left(1+4 c^{2}\right)\left\{\frac{\delta_{i j}}{1+|x|^{2}}-\frac{x^{i} x^{j}}{\left(1+|x|^{2}\right)^{2}}\right\}+\frac{12 c c_{x^{i}} c_{x^{j}}}{1+4 c^{2}} .
$$

Let

$$
f:=\frac{2 c \sqrt{1+|x|^{2}}}{\sqrt{1+4 c^{2}}}
$$

Equation (53) simplifies to $f_{x^{i} x^{j}}=0$. We obtain that $f=\epsilon+\langle a, x\rangle$. Then we obtain

$$
c=\frac{\epsilon+\langle a, x\rangle}{2 \sqrt{1+|x|^{2}-(\epsilon+\langle a, x\rangle)^{2}}} .
$$

Thus

$$
\beta=\frac{(\epsilon+\langle a, x\rangle)\langle x, y\rangle-\left(1+|x|^{2}\right)\langle a, y\rangle}{\left(1+|x|^{2}\right) \sqrt{1+|x|^{2}-(\epsilon+\langle a, x\rangle)^{2}}} .
$$

and

$$
F=\frac{\sqrt{|y|^{2}+\left(|x|^{2}|y|^{2}-\langle x, y\rangle^{2}\right)}}{1+|x|^{2}}+\frac{(\epsilon+\langle a, x\rangle)\langle x, y\rangle-\left(1+|x|^{2}\right)\langle a, y\rangle}{\left(1+|x|^{2}\right) \sqrt{\left(1+|x|^{2}\right)-(\epsilon+\langle a, x\rangle)^{2}}} .
$$

By a direct computation,

$$
1-\|\beta\|_{\alpha}^{2}=\frac{\left(1+|x|^{2}\right)\left\{1-\epsilon^{2}-|a|^{2}\right\}}{1+|x|^{2}-(\epsilon+\langle a, x\rangle)^{2}} .
$$

Thus $F=\alpha+\beta$ is a Randers metric on some open subset of $\mathrm{R}^{n}$ if and only if $\epsilon^{2}+|a|^{2}<1$. In this case, $1+|x|^{2}-(\epsilon+\langle a, x\rangle)^{2}>0$ for all $x \in \mathrm{R}^{n}$. Thus $F$ can extended to the whole $\mathrm{R}^{n}$. By (40), we obtain

$$
\mathbf{K}=\frac{3\left(1+|x|^{2}\right)}{4\left\{1+|x|^{2}-(\epsilon+\langle a, x\rangle)^{2}\right\}} \cdot \frac{F(x,-y)}{F(x, y)}+\frac{1}{4}>\frac{1}{4} .
$$

From Theorem [1.3 we obtain some interesting projectively flat Randers metrics with isotropic S-curvature.

Example 4.1 Let

$F_{-}(x, y):=\frac{\sqrt{\left(1-|x|^{2}\right)|y|^{2}+\langle x, y\rangle^{2}} \sqrt{\left(1-|x|^{2}\right)+\lambda^{2}}+\lambda\langle x, y\rangle}{\left(1-|x|^{2}\right) \sqrt{\left(1-|x|^{2}\right)+\lambda^{2}}}, \quad y \in T_{x} \mathrm{~B}^{n}$

where $\lambda \in \mathrm{R}^{n}$ is an arbitrary constant. The geodesics of $F_{-}$are straight lines in $\mathrm{B}^{n}$. Thus $F$ is of scalar curvature. One can easily verify that $F_{-}$is complete in the sense that every unit speed geodesic of $F_{-}$is defined on $(-\infty, \infty)$. Moreover $F_{-}$has strictly negative flag curvature $\mathbf{K} \leq-\frac{1}{4}$. 
Example 4.2 Let

$$
F_{0}(x, y):=\frac{|y| \sqrt{1+|x|^{2}}+\langle x, y\rangle}{\sqrt{1+|x|^{2}}}, \quad y \in T_{x} \mathrm{R}^{n} .
$$

The geodesics of $F_{0}$ are straight lines in $\mathrm{R}^{n}$. Thus $F$ is of scalar curvature. One can easily verify that $F_{0}$ is positively complete in the sense that every unit speed geodesic of $F_{0}$ is defined on $(a, \infty)$ for some $a \in \mathrm{R}$. Moreover $F_{0}$ has positive flag curvature $\mathbf{K}>0$.

\section{$5 \quad$ Proof of Theorem 1.4}

In Theorem 1.4 the manifold $M$ is compact. Assume that $\mu+4 c^{2}(x) \neq 0$ on some open subset of $M$.

When $\mu \neq 0$, let

$$
f(x):=\frac{2 c(x)}{\sqrt{ \pm\left(\mu+4 c(x)^{2}\right)}},
$$

where the sign is chosen so that $\pm\left(\mu+4 c^{2}\right)>0$. We have

$$
f_{|i| j}=-\mu f a_{i j} .
$$

This gives

$$
\Delta f=-n \mu f
$$

When $\mu=0$, we take

$$
f(x):=\frac{1}{c(x)^{2}} .
$$

We have

$$
f_{|i| j}=8 a_{i j} .
$$

This gives

$$
\Delta f=8 n
$$

Case 1: $\mu=-1$. Suppose that $1-4 c(x)^{2} \neq 0$ on $M$. Integrating (54) yields

$$
\int_{M}|\nabla f|^{2} d V_{\alpha}=-\int_{M} f \Delta f d V_{\alpha}=-n \int_{M} f^{2} d V_{\alpha}
$$

Thus $f=0$. This implies that $c=0$ and $F=\alpha$ is Riemannian.

Suppose that $1-4 c\left(x_{o}\right)^{2} \neq 0$ at some point $x_{o} \in M$. Let $\left(\tilde{M}, \tilde{x}_{o}\right)$ be the universal cover of $\left(M, x_{o}\right)$. We may assume that $\tilde{M}$ is isometric to $\left(\mathrm{B}^{n}, \alpha_{-1}\right)$ with $\tilde{x}_{o}$ corresponding to the origin. The Randers metric $F$ is lifted to a complete Randers metric $\tilde{F}$ on $\tilde{M}=\mathrm{B}^{n} . \tilde{F}$ is given by (48). Let $\tilde{c}(\tilde{x})$ be the lift of $c(x)$, which is given by (47). Thus $1-4 \tilde{c}(\tilde{x})^{2} \neq 0$ for all $\tilde{x} \in \mathrm{B}^{n}$. This implies that $1-4 c(x)^{2} \neq 0$ for all $x \in M$. By the above argument, we see that $c=0$. Hence $F=\alpha$ is Riemannian by (10). 
Suppose that $1-4 c(x)^{2} \equiv 0$. Then the lift $\tilde{F}$ of $F$ to the universal cover $\tilde{M}=\mathrm{B}^{n}$ is given by (9), hence it is incomplete. This is impossible because of the compactness of $M$. We also see that $F$ has negative constant flag curvature and bounded Cartan torsion, hence it is Riemannian according to Akbar-Zadeh's theorem [1] 28] 29. Then $c(x)=0$. This is a contradiction again.

Case 2: $\mu=0$. Suppose that $c\left(x_{o}\right) \neq 0$. Let $\tilde{M}$ denote the universal cover of $M$. We may assume that $\tilde{M}=\mathrm{R}^{n}$ with the origin corresponding to $x_{o}$. The Randers metric $F$ lifted to $\tilde{M}=\mathrm{R}^{n}$ is given by (52). Thus $c(x) \neq 0$ for all $x \in M$. Integrating (55) over $M$ yields

$$
0=\int_{M} \Delta f d V_{\alpha}=8 n \operatorname{Vol}(M, \alpha) .
$$

This is impossible. Therefore $c(x) \equiv 0$. In this case, $F$ is a locally projectively flat Randers metric with flag curvature $\mathbf{K}=0$, hence it is locally Minkowskian by [25].

Case 3: $\mu=1$. Note that $1+4 c(x)^{2} \neq 0$ on $M$. Let

$$
f(x):=\frac{2 c(x)}{\sqrt{1+4 c(x)^{2}}} .
$$

It follows from (54) that

$$
f_{|i| j}=-f a_{i j} .
$$

This gives

$$
\Delta f=-n f .
$$

Thus $f$ is an eigenfunction of $(M, \alpha)$ with $\max _{x \in M}|f|(x)<1$. We can express

$$
\begin{gathered}
F(x, y)=\alpha(x, y)-\frac{2 c_{x^{k}}(x) y^{k}}{1+4 c(x)^{2}}=\alpha(x, y)-\frac{f_{x^{k}}(x) y^{k}}{\sqrt{1-f(x)^{2}}} . \\
\mathbf{K}(x, y)=3\left\{\frac{c_{x^{k}}(x) y^{k}}{F(x, y)}+c(x)^{2}\right\}+1=\frac{3}{4\left(1-f(x)^{2}\right)} \frac{F(x,-y)}{F(x, y)}+\frac{1}{4} .
\end{gathered}
$$

Using (56), one can verify that

$$
\delta:=\sqrt{|\nabla f|_{\alpha}^{2}(x)+f(x)^{2}}
$$

is a constant. Since $F$ is positive definite, $\delta<1$.

Let

$$
\lambda(x):=\sup _{y \in T_{x} M} \frac{F(x,-y)}{F(x, y)} .
$$

Using $|\nabla f|_{\alpha}^{2}(x)=\delta^{2}-f(x)^{2}$, we obtain

$$
\lambda(x)=\frac{\sqrt{1-f(x)^{2}}+\sqrt{\delta^{2}-f(x)^{2}}}{\sqrt{1-f(x)^{2}}-\sqrt{\delta^{2}-f(x)^{2}}} .
$$


Let $\lambda:=\max _{x \in M} \lambda(x)$. We have

$$
1 \leq \lambda(x) \leq \lambda=\frac{1+\delta}{1-\delta}
$$

and

$$
1-f(x)^{2}=\frac{\left(1-\delta^{2}\right)(\lambda(x)+1)^{2}}{4 \lambda(x)} .
$$

Note that $\lambda(x)=\lambda$ if and only if $f(x)=0$. It follows from (58) that

$$
\frac{2-\delta}{2(1+\delta)}=\frac{3+\lambda}{4 \lambda} \leq \mathbf{K} \leq \frac{3 \lambda+1}{4}=\frac{2+\delta}{2(1-\delta)}
$$

Let

$$
h(x):=\arctan (2 c(x)) .
$$

The Randers metric $F(x, y)$ in (57) can be expressed by

$$
F(x, y)=\alpha(x, y)-h_{x^{k}}(x) y^{k} .
$$

Clearly $F$ is pointwise projectively equivalent to $\alpha$, namely the geodesics of $F$ are geodesics of $\alpha$ as point sets. Let $\sigma(t)$ be a closed geodesic of $\alpha$. Observe that

$$
F(\sigma(t), \dot{\sigma}(t))=\alpha(\sigma(t), \dot{\sigma}(t))-\frac{d}{d t}[h(\sigma(t))] .
$$

By the above equation we obtain

$$
\operatorname{Length}_{F}(\sigma)=\int F(\sigma(t), \dot{\sigma}(t)) d t=\int \alpha(\sigma(t), \dot{\sigma}(t)) d t=\operatorname{Length}_{\alpha}(\sigma)
$$

Assume that $M$ is simply connected. Then $(M, \alpha)=\mathrm{S}^{n}$. Let $\sigma$ be an arbitrary great circle on $\mathrm{S}^{n}$. By (59),

$$
\operatorname{Length}_{F}(\sigma)=2 \pi \text {. }
$$

\section{References}

[1] H. Akbar-Zadeh, Sur les espaces de Finsler á courbures sectionnelles constantes, Bull. Acad. Roy. Bel. Cl, Sci, 5e Série - Tome LXXXIV (1988), 281-322.

[2] S. Bácsó and M. Matsumoto, On Finsler spaces of Douglas type. A generalization of the notion of Berwald space, Publ. Math. Debrecen, 51(1997), 385-406.

[3] D. Bao, S. S. Chern and Z. Shen, An Introduction to Riemann-Finsler Geometry, Springer-Verlag, 2000. 
[4] D. Bao and C. Robles, On Randers metrics of constant curvature, Reports on Mathematical Physics (to appear).

[5] D. Bao, C. Robles and Z. Shen, Zermelo Navigation on Riemannian manifolds, preprint.

[6] D. Bao and Z. Shen, Finsler metrics of constant curvature on the Lie group $S^{3}$, J. of London Math. Soc. 66(2002), 453-467.

[7] L. Berwald, Untersuchung der Krümmung allgemeiner metrischer Räume auf Grund des in ihnen herrschenden Parallelismus, Math. Z. 25(1926), 40-73.

[8] L. Berwald, Parallelübertragung in allgemeinen Räumen, Atti Congr. Intern. Mat. Bologna 4(1928), 263-270.

[9] R. Bryant, Finsler structures on the 2-sphere satisfying $K=1$, Finsler Geometry, Contemporary Mathematics 196, Amer. Math. Soc., Providence, RI, 1996, 27-42.

[10] R. Bryant, Projectively flat Finsler 2-spheres of constant curvature, Selecta Math., New Series, 3(1997), 161-204.

[11] R. Bryant, Some remarks on Finsler manifolds with constant flag curvature, Houston J. of Math. 28(2002), 221-262.

[12] X. Chen and Z. Shen, Randers Metrics with Special Curvature Properties, Osaka J. of Math., (to appear).

[13] S. S. Chern, On the Euclidean connections in a Finsler space, Proc. National Acad. Soc., 29(1943), 33-37; or Selected Papers, vol. II, 107111, Springer 1989.

[14] A. Deicke, Über die Finsler-Raume mit $A_{i}=0$, Arch. Math. 4(1953), $45-51$.

[15] P. Funk, Über Geometrien, bei denen die Geraden die Kürzesten sind, Math. Annalen 101(1929), 226-237.

[16] P. Funk, Über zweidimensionale Finslersche Räume, insbesondere über solche mit geradlinigen Extremalen und positiver konstanter Krümmung, Math. Zeitschr. 40(1936), 86-93.

[17] P. Funk, Eine Kennzeichnung der zweidimensionalen elliptischen Geometrie, Österreichische Akad. der Wiss. Math., Sitzungsberichte Abteilung II 172(1963), 251-269.

[18] D. Hilbert, Mathematical Problems, Bull. of Amer. Math. Soc. 37(2001), 407-436. Reprinted from Bull. Amer. Math. Soc. 8 (July 1902), 437-479. 
[19] M. Matsumoto, Foundations of Finsler Geometry and special Finsler Spaces, Kaiseisha Press, Japan 1986.

[20] M. Matsumoto and H. Shimada, The corrected fundamental theorem on the Randers spaces of constant curvature, Tensor, N.S. (to appear).

[21] X. Mo, The flag curvature tensor on a closed Finsler space, Results in Math. 36(1999), 149-159.

[22] X. Mo, On the flag curvature of a Finsler space with constant Scurvature, preprint.

[23] X. Mo and Z. Shen, On negatively curved Finsler manifolds of scalar curvature, preprint.

[24] Z. Shen, Differential Geometry of Spray and Finsler Spaces, Kluwer Academic Publishers, Dordrecht, 2001.

[25] Z. Shen, Lectures on Finsler Geometry, World Scientific, Singapore (2001), 307 pages.

[26] Z. Shen, Projectively flat Randers metrics with constant flag curvature, Math. Ann. 325(2003), 19-30.

[27] Z. Shen, Projectively flat Finsler metrics of constant flag curvature, Trans. of Amer. Math. Soc. 255(2003), 1713-1728.

[28] Z. Shen, Finsler metrics with $\mathbf{K}=0$ and $\mathbf{S}=0$, Canadian J. Math. $\mathbf{5 5}(2003), 112-132$.

[29] Z. Shen, Two-dimensional Finsler metrics of constant curvature, Manuscripta Mathematica 109(2002), 349-366.

[30] E. Zermelo, Über das Navigationsproblem bei ruhender oder veränderlicher Windverteilung, Z. argrew. Math. Mech. 11(1931), 114124.

Xinyue Chen

Department of Mathematics, Chongqing Institute of Technology, Chongqing 400050, P.R. China

chenxy58@163.net

Xiaohuan Mo

LMAM, School of Mathematical Sciences, Beijing University, Beijing 100871, P.R. China moxh@pku.edu.cn

Zhongmin Shen

Department of Mathematical Sciences, Indiana University-Purdue University Indianapolis, 402 N. Blackford Street, Indianapolis, IN 46202-3216, USA.

zshen@math.iupui.edu 